\title{
Head-related transfer functions of the Mongolian gerbil in the median plane
}

\author{
Katuhiro Maki, Shigeto Furukawa and Tatsuya Hirahara \\ Human and Information Science Laboratory, NTT Communication Science Laboratories, NTT Corporation, \\ 3-1 Morinosato-Wakamiya, Atsugi, Kanagawa 243-0198, Japan
}

(Received 3 February 2003, Accepted for publication 20 February 2003)

Keywords: HRF, DF, Sound localization, Pinna, Outer ear PACS number: 43.64.Ha, 43.66.Qp [DOI: 10.1250/ast.24.330]

\section{Introduction}

Mongolian gerbils are widely used in physiological and behavioral experiments on sound source localization [1-4]. For small mammals like the gerbils, sound source localization is important for survival in their natural environment because sound source information helps them understand the location of prey and predators. However, it is unclear whether the sound-localization cues for small mammals are the same type as those for larger mammals, such as cats, monkeys, and humans. This is because they have much smaller heads and a markedly different pinna shape. This study investigated the head-related transfer functions (HRTFs) of a gerbil in the median plane. We surgically placed a probe-tube microphone into the ear canal, and measured the HRTFs at various speaker locations in elevation.

\section{Methods}

The experiments were performed on one male Mongolian gerbil (Meriones unguiculatus), weighing $74 \mathrm{~g}$. A small incision was made at the ventro-posterior end of the pinna, through which a polyethylene tube $(2.5 \mathrm{~cm}$ length, $0.6 \mathrm{~mm}$ inner diameter, $1.0 \mathrm{~mm}$ outer diameter) was inserted (Fig. 1a).

A small hole was made on the wall of the bony meatus to fix the tip of the tube, so that the entrance of the tube in the ear was located $<0.5 \mathrm{~mm}$ from the surface of the upper half of the tympanic membrane. The other end of the tube was connected to a probe tube microphone (Brüel and Kjær, type 4182). The animal body was secured by fixing the waist and the chest of the animal using a custom-made wire frame so that the animal's posture was close to its natural standing posture. The animal's head was positioned at the center of the imaginary sphere made by the trajectory of the sound source.

A movable speaker system was used for sound presentation (Fig. 1b). The system consisted of a semi-circular arm (3/ 4 circular arc, $1.5 \mathrm{~mm}$ diameter), along which a sound source moved as driven by a stepping motor. The system allowed us to vary the sound source location on the animal's median plane. We represent the sound source location in elevation angle relative to the horizontal plane that includes the interaural axis; $0^{\circ}$ elevation refers to the source straight in front of the animal; $90^{\circ}$ elevation refers to the source directly overhead. A time-stretched-pulse (TSP) [5], was synthesized digitally by a personal computer (PC) at a sampling rate of $100 \mathrm{kHz}$ with a resolution of 16 bits. Total points of the TSP signal were $65536\left(2^{16}\right)$. The TSP signal was generated through a D/A converter (Tucker-Davis Tech., RP 2.1), amplified (Nittobo Acoust. Eng., HA-94C), and played from a tweeter (Sony, SS-TW100ED).

Experiments were performed in an anechoic room (W $4.8 \mathrm{~m} \times \mathrm{L} 5.4 \mathrm{~m} \times \mathrm{H} 4.7 \mathrm{~m}$ ) at NTT Atsugi R\&D Center. The output of the probe microphone was amplified (Brüel and Kjær, type 2636) and stored on the hard disk of a PC through the sound control system (Tucker-Davis Tech., RP 2.1) at a sampling rate of $100 \mathrm{kHz}$ with a resolution of 16 bits. Recorded signals from fifty repetitions were averaged in the time domain to increase the signal to noise ratio. Prior to the measurements from the animal, the frequency response of the speaker-microphone system was measured using the microphone with the extension polyethylene tube located at the center of the interaural axis of the animal.

We calculated the impulse response from a recorded TSP signal based on the time-stretched-pulse theory [5]. Each impulse response was truncated to 512 points. We calculated the frequency response from the impulse response using 512point FFT. We derived the head-related transfer function (HRTF) from a sound source to a point near the tympanic membrane by dividing the frequency response derived from the measurement from the animal's ear by the frequency response of the speaker-microphone system [6,7]. We also calculated directional transfer functions (DTFs), which were obtained by dividing the HRTFs for each location by the HRTF averaged across all measured locations. The DTFs represent the source-direction dependent components extracted from the HRTFs [8].

\section{Results and discussion}

Figure 2a shows an example of the HRTFs for one ear of the animal. The figure represents source elevations ranging from $-40^{\circ}$ to $220^{\circ}$.

In Fig. 2a, one can see marked spectral features at frequencies above $5 \mathrm{kHz}$. In general, maximal gains are found in the frequency range between 10 and $20 \mathrm{kHz}$, the peak being about $10-15 \mathrm{~dB}$ above the gain at $10 \mathrm{kHz}$. The peak frequency was essentially independent of the source elevation. These elevation-independent peaks are due to the resonance of the ear canal [9]. Above $20 \mathrm{kHz}$, one can see distinct features that varied with the elevation locations. These elevation-dependent spectral features are more apparent in the DTFs (Fig. 2b). Specifically, a deep spectral notch (arrow) shifted systematically from about 25 to $40 \mathrm{kHz}$ as the speaker location moved from $-40^{\circ}$ to $80^{\circ}$. Figure 3 shows the notch frequency as a function of elevation. Here, we define the notch frequency as 


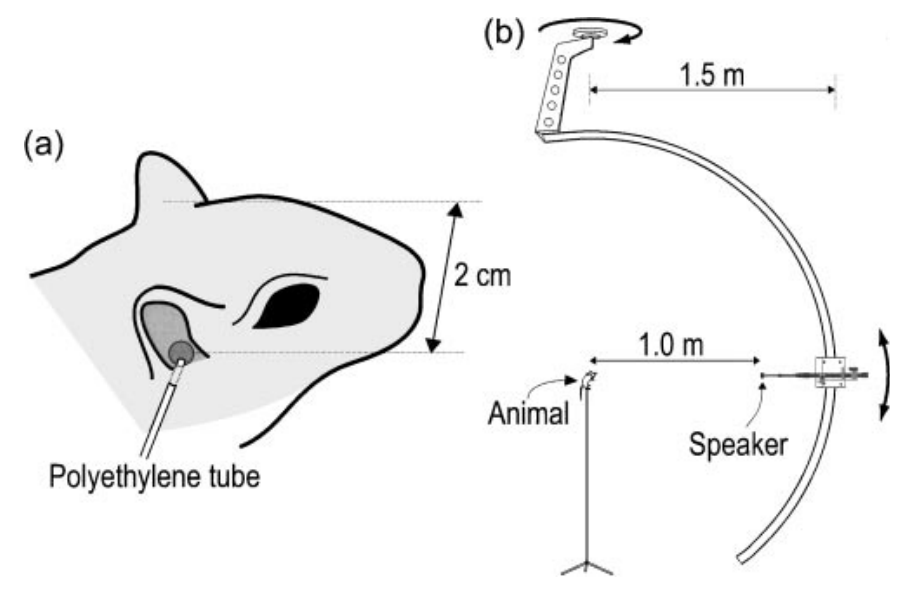

Fig. 1 Microphone setup and measurement system. (a) A polyethylene tube for connecting the microphone was inserted into the auditory meatus near the tympanic membrane though the ventro-posterior end of the pinna. (b) A movable speaker system for presenting a sound for the animal, which allowed the speaker to move from $-40^{\circ}$ to $220^{\circ}$ elevation locations.
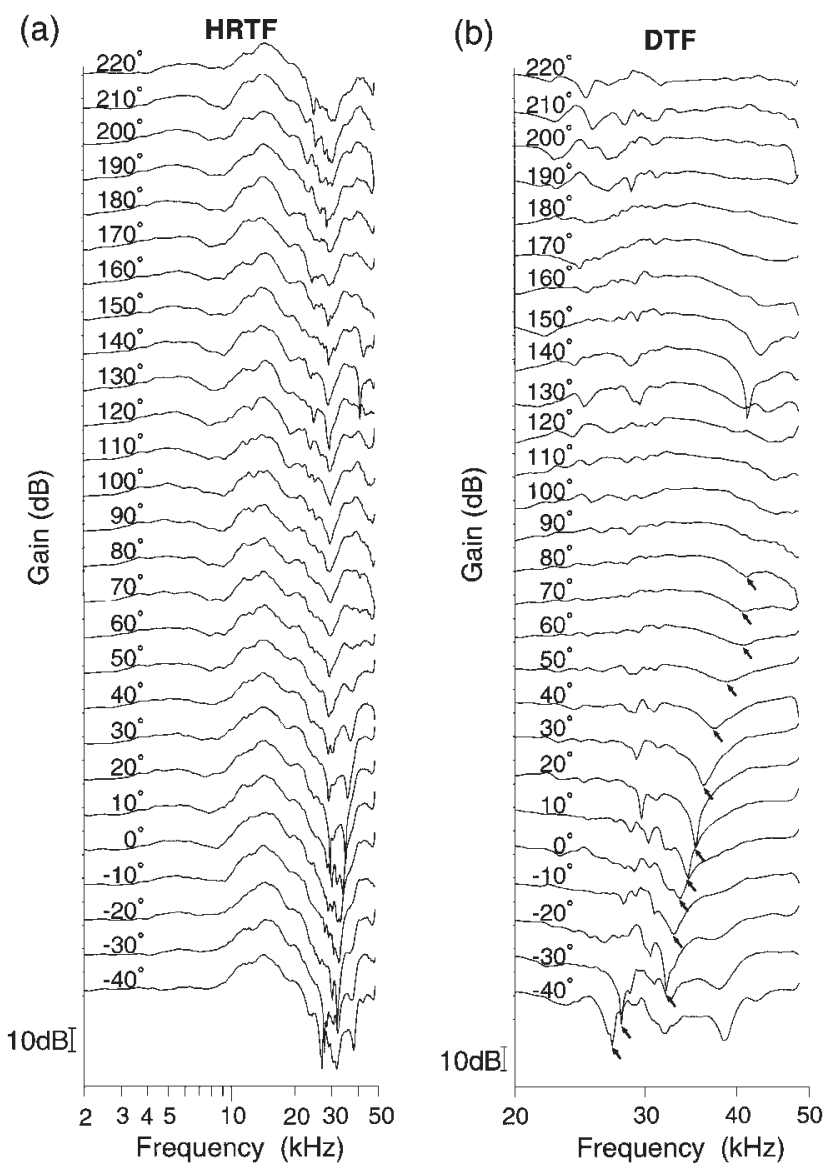

Fig. 2 The head-related transfer functions (HRTFs) and directional transfer functions (DTFs) across elevation locations from one ear of the animal. (a) HRTFs for the 27 elevation locations in the vertical midline plane (azimuth $=0^{\circ}$ ). Elevation locations are shown in the left figure for each HRTF curve. (b) DTFs calculated by HRTFs shown in (a) (See Methods in the text).

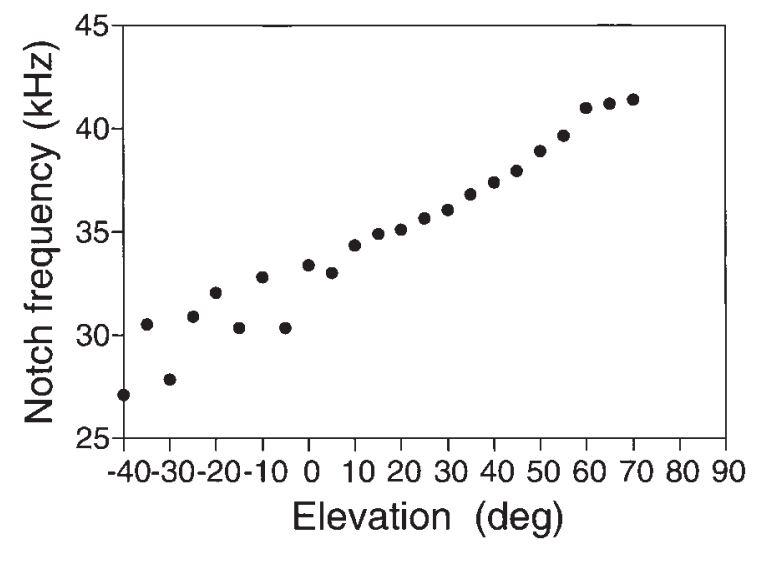

Fig. 3 The elevation dependence of notch frequency above $20 \mathrm{kHz}$ for locations at $0^{\circ}$ azimuth at the various elevations. Frequencies of minimal gain were detected as notch frequencies from the DTFs shown in Fig. 2b.

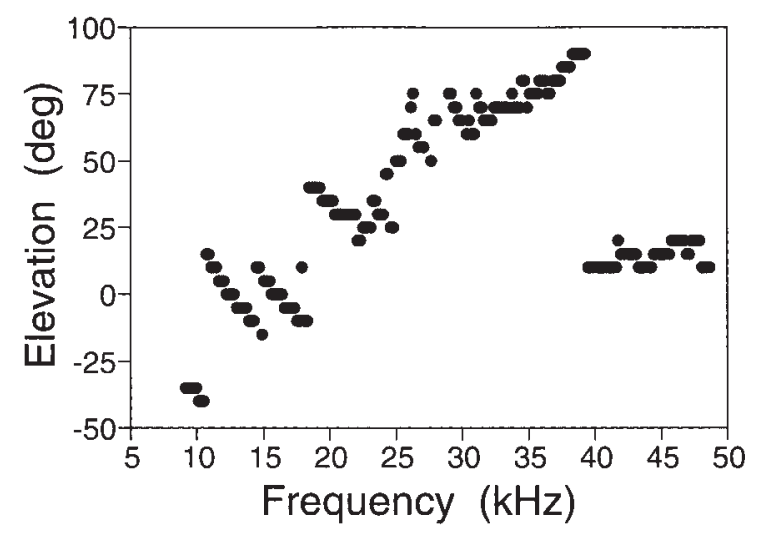

Fig. 4 The acoustic axis as a function of frequency from 5 to $50 \mathrm{kHz}$. The acoustic axis is defined for each frequency as the direction of maximal gain for that particular frequency. This acoustic axis is calculated from the data shown in Fig. 2.

the frequency that has minimum gain in the DTF for frequencies above $20 \mathrm{kHz}$. Figure 3 shows that the notch frequency basically increased monotonically with increasing speaker elevation.

Previous studies used the "acoustic axis" as a measure of the directionality of the pinna and ear canal [6,10-12]. The acoustic axis is defined for each frequency as the direction of maximal gain for that particular frequency.

One trend shown in Fig. 4 is that the acoustic axis changes from lower to higher elevations as the frequency increases from 9 to $39 \mathrm{kHz}$. Around $39 \mathrm{kHz}$, there was an abrupt shift in elevational locations from $90^{\circ}$ to $10^{\circ}$.

The HRTFs of the gerbil in the present study showed spectral features, such as peaks and notches, in the frequency range above $20 \mathrm{kHz}$, all of which varied somewhat systematically with the sound source elevation. Specifically, the spectral-notch frequency of the DTFs tended to increase with the sound source elevation (Fig. 3). Also, the acoustic axis generally increased with increasing frequency, with abrupt transitions at certain frequencies (Fig. 4). Similar patterns of 
Table 1 Acoustical parameters of HRTFs of gerbil and other mammalian species. Parameter values of the gerbil are from our experiments, except for audible frequency range data [16]. Head diameters of cat and monkey are estimated from interaural time differences measured for the animals $[13,17]$. Audible frequency range data are from $[16,18]$, the other data are from $[6-8,13-15]$.

\begin{tabular}{lcccc}
\hline & Gerbil & Cat & Rhesus monkey & Human \\
\hline Head diameter & $\approx 2 \mathrm{~cm}$ & $\approx 8 \mathrm{~cm}$ & $\approx 11 \mathrm{~cm}$ & $\approx 13 \mathrm{~cm}$ \\
Audible frequency range & $0.25-45 \mathrm{kHz}$ & $0.125-60 \mathrm{kHz}$ & $0.125-32 \mathrm{kHz}$ & $0.13-16 \mathrm{kHz}$ \\
Frequency of maximal gain & $13-17 \mathrm{kHz}$ & $3-5 \mathrm{kHz}$ & $4-5 \mathrm{kHz}$ & $2.5-4 \mathrm{kHz}$ \\
Frequency range of elevation-dependent & $27-42 \mathrm{kHz}$ & $8-16 \mathrm{kHz}$ & $5-15 \mathrm{kHz}$ & $6-12 \mathrm{kHz}$ \\
spectral notches & & & \\
\hline
\end{tabular}

correspondence between the spectral features and the source elevation have been observed in other mammalian species (guinea pigs [10], wallabies [12], cats [6-8], Rhesus monkeys [13]) as well as humans $[14,15]$. A major difference among species is the frequency range of elevation-dependent spectral features.

Table 1 compares the frequency range of the elevationdependent spectral notches, for the gerbil, cat, Rhesus monkey, and human. It also shows head diameters, audible frequency ranges, and frequency ranges of maximal gain. One can see that the frequency range of the elevation-dependent spectral notches varies markedly across species, which we consider to be due largely to differences of the size of the head and pinna. Among the species shown, the frequency range of the elevation-dependent spectral notches is highest in the gerbil: The frequency range for the gerbil was as high as $42 \mathrm{kHz}$, about 3-4 times higher than that for humans. We should note, however, that the gerbil's audible frequency range extends up to $45 \mathrm{kHz}$, which covers the frequency range for the spectral notches, indicating that the gerbil could take advantage of the DTF information in identifying sound source elevation.

\section{Conclusion}

We investigated the HRTFs of a gerbil. The results showed that the spectral features in the HRTFs, e.g., the elevation-dependent spectral notches, varied somewhat systematically with the sound source elevation, in such a way that was consistent with the data obtained for larger mammalian species, including humans. The results suggests that the gerbil could be used as an animal model for physiological and behavioral studies of human sound-source localization.

\section{Acknowledgements}

We thank the Interdepartmental Laboratories of Tokai University for assistance in animal care for the experiments.

\section{References}

[1] S. Bruckner and R. Rubsamen, "Binaural response characteristics in isofrequency sheets of the gerbil inferior colliculus," Hear. Res., 86, 1-14 (1995).

[2] M. W. Spitzer and M. N. Semple, "Interaural phase coding in auditory midbrain: influence of dynamic stimulus features," Science, 254, 721-724 (1991).

[3] R. S. Heffner and H. E. Heffner, "Sound localization and use of binaural cues by the gerbil, (Meriones unguiculatus)," Behav. Neurosci., 102, 422-428 (1988).
[4] J. B. Kelly and M. Potash, "Directional responses to sounds in young gerbils, (Meriones unguiculatus)," J. Comp. Psychol., 100, 37-45 (1986).

[5] Y. Suzuki, F. Asano, H.-Y. Kim and T. Sone, "An optimum computer-generated pulse signal suitable for the measurement of very long impulse responses," J. Acoust. Soc. Am., 97, 1119-1123 (1995).

[6] A. D. Musicant, J. C. Chan and J. E. Hind, "Directiondependent spectral properties of cat external ear: new data and cross-species comparisons," J. Acoust. Soc. Am., 87, 757-781 (1990).

[7] J. J. Rice, G. A. Spirou and E. D. Young, "Pinna-based spectral cues for sound localization in cat," Hear. Res., 58, 132-152 (1992).

[8] L. Xu and J. C. Middlebrooks, "Individual differences in external-ear transfer functions of cats," J. Acoust. Soc. Am., 107, 1451-1459 (2000).

[9] M. E. Ravicz, J. J. Rosowski and H. F. Voigt, "Sound-power collection by the auditory periphery of the Mongolian gerbil Meriones unguiculatus. II. External-ear radiation impedance and power collection," J. Acoust. Soc. Am., 99, 3044-3063 (1996).

[10] S. Carlile and A. G. Pettigrew, "Directional properties of the auditory periphery in the guinea pig," Hear. Res., 31, 111-122 (1987).

[11] P. H. Jen and D. M. Chen, "Directionality of sound pressure transformation at the pinna of echolocating bats," Hear. Res., 34, 101-117 (1988).

[12] R. B. Coles and A. Guppy, "Biophysical aspects of directional hearing in the Tammar Wallaby, Macropus eugenii," J. Exp. Biol., 121, 371-394 (1986).

[13] M. L. Spezio, C. H. Keller and T. T. Takahashi, "Head-related transfer functions of the Rhesus monkey," Hear. Res., 144, 7388 (2000).

[14] J. C. Middlebrooks, "Individual differences in external-ear transfer functions reduced by scaling in frequency," J. Acoust. Soc. Am., 106, 1480-1492 (1999).

[15] H. Møller, M. F. Sørensen, D. Hammershøi and C. B. Jensen, "Head-related transfer functions of human subjects," J. Audio. Eng. Soc., 43, 300-320 (1995).

[16] S. M. Echteler, R. R. Fay and A. N. Popper, "Structure of the Mammalian Cochlea," in Comparative Hearing: Mammals, R. R. Fay, A. N. Popper, Eds. (Springer-Verlag, New York, 1994), Chap. 5, pp. 134-171.

[17] G. L. Roth, R. K. Kochhar and J. E. Hind, "Interaural time differences: implications regarding the neurophysiology of sound localization," J. Acoust. Soc. Am., 68, 1643-1651 (1980).

[18] B. E. Pfingst, J. Laycock, F. Flammino, B. Lonsbury-Martin and G. Martin, "Pure tone thresholds for the rhesus monkey," Hear. Res., 1, 43-47 (1978). 